\title{
One-way absorption of terahertz waves in rod-type and multilayer structures containing polar dielectrics
}

\author{
Andriy E. Serebryannikov, ${ }^{1,2,3,{ }^{*}}$ Shunji Nojima, ${ }^{4}$ and Ekmel Ozbay ${ }^{2}$ \\ ${ }^{1}$ Faculty of Physics, Adam Mickiewicz University, 61-614 Poznań, Poland \\ ${ }^{2}$ Nanotechnology Research Center - NANOTAM, Bilkent University, 06800 Ankara, Turkey \\ ${ }^{3}$ Department of Electrical and Electronics Engineering, Universidad Pública de Navarra, 31006 Pamplona, Spain \\ ${ }^{4}$ Yokohama City University, Department of Nanosystem Science, Graduate School of Nanobioscience, \\ Kanazawa Ku, Yokohama, Kanagawa 2360027, Japan
}

(Received 14 May 2014; revised manuscript received 26 November 2014; published 16 December 2014)

\begin{abstract}
One-way absorption can be obtained at terahertz frequencies in low-profile rod-type and multilayer dielectric structures with broken spatial inversion symmetry, which contain either a rod layer or an ultrathin homogeneous layer made of a polar dielectric. Perfect absorption for one of the two opposite incidence directions and perfect reflection for the other one are observed at the edge of the polaritonic gap in a wide range of the incident angle variation, when the thickness of the entire structure is of the order of the incident wavelength. Moreover, this regime appears in a wide frequency range, in which the forward-to-backward absorption contrast is strong. The exploited mechanism is connected with the parameter adjustment that enables the location of the polaritonic gap of the polar dielectric, of which the lossy part of the structure is made, inside the stop band arising due to the periodicity of the lossless part of the structure that is made of a nondispersive dielectric. It also exploits absorption enhancement in the lossy part by backing it with the highly reflecting lossless part, which has alternating stop and pass bands.
\end{abstract}

DOI: 10.1103/PhysRevB.90.235126

PACS number(s): 42.25.Bs, 42.70.Qs, 71.36.+c, 78.67.Pt

\section{INTRODUCTION}

In recent years, the interest in the asymmetric effects arising in the Lorentz reciprocal structures with broken spatial inversion symmetry has been growing [1-5]. Several mechanisms of asymmetric transmission have been suggested, whose common feature is that the additional transmission and reflection channels must be open at one of the two opposite incidence directions. In other words, they cannot be realized in a two-port system that is made of linear, isotropic, and passive materials. The known mechanisms include those inspired by diffractions [1,2,6,7] and polarization conversion [5,8,9]. The mechanisms realizable in the structures that (may) support surface waves should also be mentioned [10-12]. Clearly, full analogs of nonreciprocal effects cannot be achieved in the framework of these mechanisms [13]. In contrast to transmission, asymmetry in reflection and absorption does not require additional channels. It can appear in a two-port system, provided that spatial inversion symmetry is broken and at least one of the two structural components is lossy [14]. In particular, asymmetry in reflection has been demonstrated in Refs. $[15,16]$. The simplest case, in which the forward-case reflectance and absorbance differ from the backward-case ones, is expected to be a two-layer structure with one lossy layer. However, the obtaining of high forward-to-backward contrasts in low-profile structures remains a challenging task.

Among the recently suggested absorbers, those based on quasiplanar metamaterials should be mentioned, which enable perfect (unity) absorption [17-23]. High-efficiency absorption can be obtained in a wide range of the incidence angle variation [24-26], including the case of perfect absorption [27-30]. Recently, the theory of resonance absorption in ultrathin

\footnotetext{
*andser@amu.edu.pl
}

structures has been developed and implemented [31-33]. In particular, the possibility of using the perfectly absorbing layers being as thin as 5-20 $\mathrm{nm}$ has been demonstrated. Although these absorbers are thin (that is a very important advantage), the question remains as to whether they represent the topologically simplest configurations for such operation regimes. To the best of our knowledge, the possibility of asymmetric absorption in the mentioned thin absorbers, although being possible, has not yet been considered.

On the other hand, structures for multifunctional operation are required, in which asymmetric absorption would co-exist with high-efficiency pass and stop bands, for most of which the effect of the losses is weak. From this perspective, designs based on two-dimensional photonic crystals (PhCs) and one-dimensional $\mathrm{PhCs}$, i.e., multilayers, are promising. Polar dielectrics are natural materials showing strong absorption within a certain terahertz frequency range and rather weak absorption beyond it. The polaritonic gap and strong dependence of permittivity on frequency belong to the basic properties of these materials, which have been utilized in various applications related to electromagnetic wave manipulation. In particular, multiple excitonic polaritons and polaritonic gaps can appear in rather simple structures, e.g., in one-dimensional PhCs [34-36]. Terahertz metamaterials containing rods made of a polar dielectric that may show hyperbolic dispersion, superlensing, near-zero and negative permeability, negative index, and other interesting effects are also worth mentioning [37-39].

In this paper, we study wide-angle, perfectly asymmetric absorption arising at terahertz frequencies in rod arrays with broken structural symmetry that represent thin fragments of two-dimensional PhCs and in multilayers, both made of conventional polar (lossy) and nondispersive (lossless) dielectrics. The general approach is based here on the parameter adjustment that enables the spectral location of the polaritonic 
gap of the polar dielectric inside the stop band arising due to the periodicity of the structural part made of nondispersive dielectric(s). The main aim is to demonstrate the possibility of an extremely strong forward-to-backward absorption contrast for nonsolid configurations, e.g., in nonsymmetric three-layer and four-layer rod arrays in air host, in which one layer is made of a polar dielectric, and the remaining layers are made of a nondispersive dielectric. Backing the lossy layer with a structure showing strong reflections in the stop band will be used to simultaneously enhance the forwardcase absorption (for the lossy layer side incidence) and backward-case reflection (for the opposite side incidence), and obtain perfect absorption in the former case. Strong directional selectivity in absorption will also be demonstrated in nonsymmetric multilayer structures made of nondispersive dielectrics that are stacked with an ultrathin polar dielectric layer. Perfect one-way absorption may appear in a wide range of variation of the incidence angle. The presented results are obtained by using the coupled integral equation technique, an efficient iterative solver with preconditioning that has controllable convergence and accuracy and is quite flexible regarding geometrical and material parameters of the studied structures [40]. Transmittance $(T)$ and reflectance $(R)$ are directly obtained from this technique, and then absorption is obtained from $A=1-T-R$.

\section{THEORETICAL BACKGROUND}

The general idea of the suggested one-way absorption mechanism is illustrated by Fig. 1(a). If a plane electromagnetic wave is incident from the side of the lossy part made of a polar dielectric, perfect absorption is expected to be possible. If the incidence direction is changed to the opposite one, perfect reflections should appear. Examples of the rod based structures that enable the suggested mechanism of one-way absorption are presented in Figs. 1(b) and 1(c). The structures are assumed to be periodic with period $a$ in the $x$ direction and infinitely extended along the rod axes, and have finite thickness in the $y$ direction. The distance between the rod layers in the $y$ direction is also equal to $a$, i.e., it represents the lattice constant. Rod diameter is denoted by $d$. The multilayer structures (see Figs. 8 and 9) also have finite thickness and infinite extent in the $x$ direction and in the direction corresponding to the rod axes in the rod type structures. We assume that $s$-polarized electromagnetic wave is incident from either the P side (forward incidence) or ND side (backward incidence). These cases are indicated by $\rightarrow$ and $\leftarrow$, respectively. Directions of incidence are assumed to be opposite, while the angle of incidence $\theta$ is measured in the counterclockwise direction with respect to the normal to the corresponding incidence interface, as shown in Figs. 1(b) and $1(\mathrm{c})$ in the forward-incidence case.

As a result of phonon-photon coupling and polariton excitation, polar dielectrics behave as strongly dispersive lossy media, whose frequency dependence of permittivity is given as follows $[34,41]$ :

$$
\epsilon_{P}(\omega)=\epsilon_{\infty}+\left(\epsilon_{0}-\epsilon_{\infty}\right) \omega_{T}^{2} /\left(\omega_{T}^{2}-\omega^{2}-i \Gamma \omega\right),
$$

where $\epsilon_{0}$ is the static permittivity, $\epsilon_{\infty}$ is the high-frequency limit of permittivity, and $\Gamma$ is the absorption factor. One of
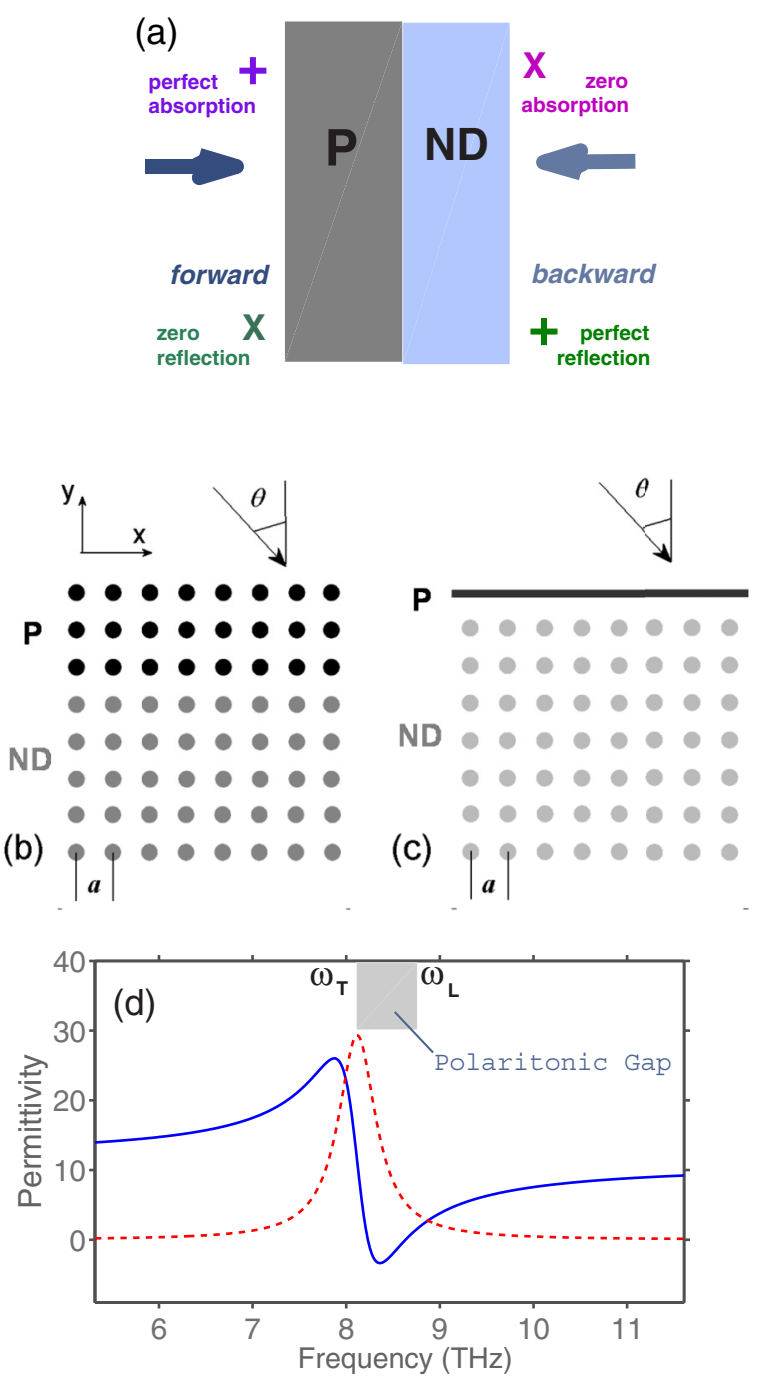

FIG. 1. (Color online) (a) Schematic illustrating perfect one-way absorption in structures with broken structural symmetry; examples of the structures composed of both polar (lossy) dielectrics and nondispersive (lossless) dielectrics in one configuration with (b) the same and (c) different shape of the components that are made of dielectrics of two types; P and ND denote the parts that are fully made of a polar dielectric and a nondispersive lossless dielectric, respectively; (d) permittivity of GaAs: $\operatorname{Re} \epsilon_{P}$, solid line, and $\operatorname{Im} \epsilon_{P}$, dashed line.

the basic features of polar dielectrics is the existence of the polaritonic gap, which corresponds to negative permittivity. Its lower and upper boundaries are commonly denoted by angular frequencies $\omega_{T}$ and $\omega_{L}$, respectively. These frequencies satisfy the Lyddane-Sachs-Teller relation [41], i.e., $\omega_{L}^{2} / \omega_{T}^{2}=$ $\epsilon_{0} / \epsilon_{\infty}$. In this paper, we use GaAs, for which $\epsilon_{\infty}=10.9$, $\omega_{T} /(2 \pi)=8.12 \mathrm{THz}, \omega_{L} /(2 \pi)=8.75 \mathrm{THz}[34,41]$, and $\Gamma / \omega_{T}=0.06$ [34]. The corresponding frequency dependence of $\epsilon_{P}$ is presented in Fig. 1(d). Note that the actual frequency interval, in which $\operatorname{Re} \epsilon_{P}<0$, is here narrower than the interval $\omega_{T}<\omega<\omega_{L}$ owing to nonzero $\Gamma$. Throughout the paper, $M$ and $N$ denote the number of the rod layers made of nondispersive and polar dielectrics, which correspond to the structural parts denoted by ND and P; the asterisk indicates 

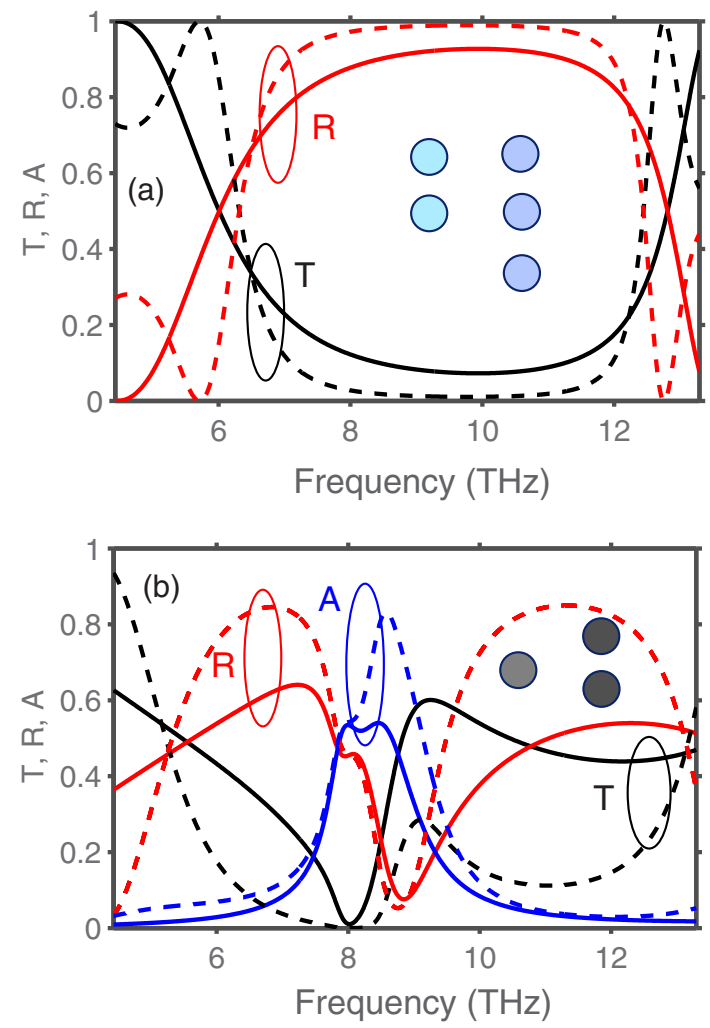

FIG. 2. (Color online) (a) $T$ and $R$ for rod arrays made of nondispersive dielectric at $M=2$ (solid line) and $M=3$ (dashed line), with $\epsilon_{\mathrm{ND}}=\epsilon_{\infty}=10.9$; (b) $T, R$, and $A$ for rod arrays made of GaAs at $N=1$ (solid line) and $N=2$ (dashed line); $d / a=0.4$, $\theta=0$; two insets (in each plot) schematically show geometry of a single period in the $x$ direction, for both structures.

perfect absorption, $A=1$; the value of $a=10.8 \mu \mathrm{m}$ is chosen so that $\omega_{T} a / c=7 \pi / 12$. The insets in all of the cases show a structure configuration within a single period over the $x$ axis.

Figure 2 presents $T, R$, and $A$ vs frequency for the singlelayer, double-layer, and triple-layer symmetric structures composed of either nondispersive (lossless) or polar (lossy) dielectric rods. These structures will further be used as the building blocks of the composite nonsymmetric structures that contain both polar and nondispersive dielectrics. In Fig. 2(a), a well pronounced stop band is observed although three layers of nondispersive dielectric rods are only used. It can be considered as a reminiscence of the lowest band gap of the corresponding two-dimensional PhC. For similar structures in Fig. 2(b), which contain the GaAs rods, strong absorption is the dominant feature in and near the polaritonic gap. However, perfect absorption, i.e., $A=1$, is not achieved in these structures. Variation of the distance between the two rod layers in Fig. 2(b) does not lead to a significant increase of $A$ (not shown).

Next, we suppose that stacking layers of the rods made of nondispersive and polar dielectrics can lead to absorption enhancement due to reflections from the nondispersive rod layers in the forward-incidence case. In terms of the expected role of reflections for absorption, the basic mechanism here is similar to what was studied in [31,32]. A composite structure should be designed in such a way that the polaritonic gap is located inside the stop band that appears due to the periodicity in the $y$ direction. As follows from the obtained results, this enhancement mechanism can work also at small values of $M$ and $N$. Moreover, breaking spatial inversion symmetry can result in strong asymmetry between the forwardcase absorption, $A^{\rightarrow} \neq 0$, and the backward-case absorption, $A^{\leftarrow}=0$. In the latter case, the incident wave should entirely be reflected in the stop band, i.e., $R^{\leftarrow}=1$.

\section{RESULTS AND DISCUSSION}

The basic features of the above described mechanism are demonstrated in Fig. 3 for the two composite structures. It is seen that $\max A^{\rightarrow}$ in the case of stacking of one nondispersive rod layer and one polar dielectric rod layer in Fig. 3(a) is nearly the same as in the case of two polar dielectric layers in Fig. 2(b). Hence, absorption enhancement is obtained without adding more rods that are made of a lossy dielectric and, thus, without increasing its total volume. A comparison of Figs. 3(a) and 3(b) indicates the existence of strong asymmetry, i.e., $A^{\rightarrow}=0.8$ and $A^{\leftarrow}=0.15$, while the reciprocity requires that $T^{\rightarrow}=T^{\leftarrow}$ and, hence, $R^{\rightarrow}+A^{\rightarrow}=R^{\leftarrow}+A^{\leftarrow}$. The increase of $M$ leads to stronger reflection from the nondispersive part of the structure and, accordingly, to the increase of $A^{\rightarrow}$ and decrease of $A^{\leftarrow}$. In Figs. 3(c) and 3(d), an example is presented, in which $A^{\rightarrow}=1$ and $A^{\leftarrow}=0$ are observed at $f \approx 8.68 \mathrm{THz}$, whereas $R^{\rightarrow}=0$ and $R^{\leftarrow}=1$. This regime appears at the upper edge of the polaritonic gap, i.e., when $\operatorname{Im} \epsilon_{P} \approx 4.6$ that is rather far from the maximal value; see Fig. 1(d). Moreover,
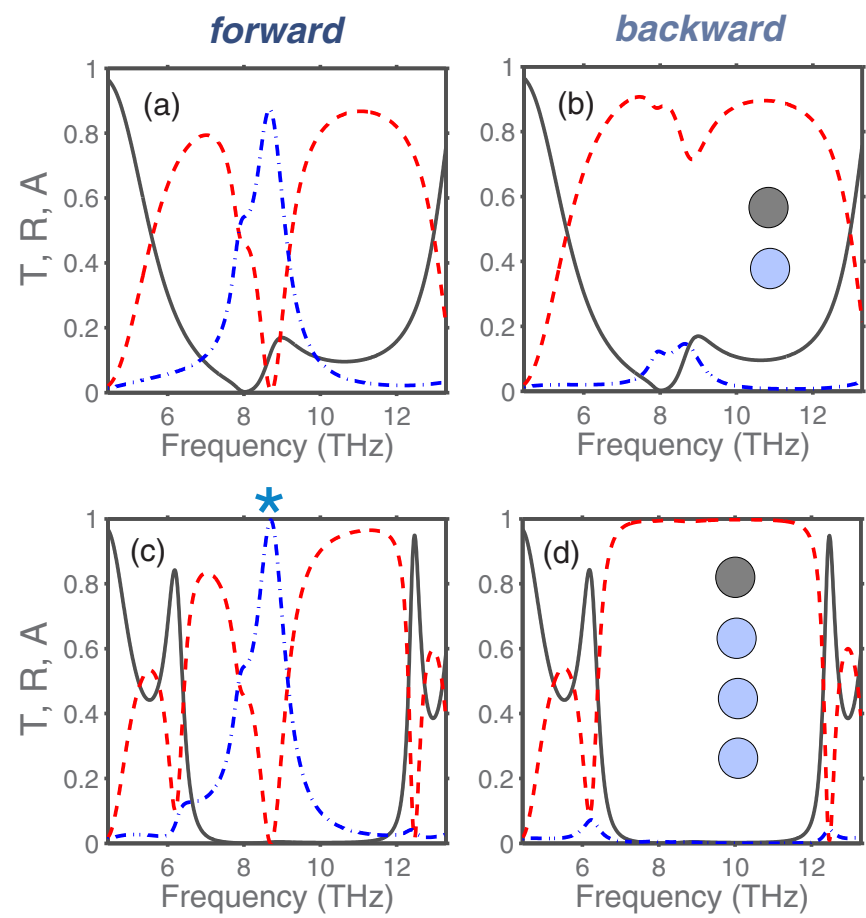

FIG. 3. (Color online) $T^{\rightarrow}, R^{\rightarrow}$, and $A^{\rightarrow}$ (a), (c) and $T^{\leftarrow}, R^{\leftarrow}$, and $A^{\leftarrow}$ (b), (d), for two composite structures with (a), (b) $M=1$ and $N=1$, and (c), (d) $M=3$ and $N=1 ; d / a=0.4, \theta=0$; solid line: $T^{\rightarrow}$ and $T^{\leftarrow}$; dashed line: $R^{\rightarrow}$ and $R^{\leftarrow}$; dash-dotted line: $A^{\rightarrow}$ and $A^{\leftarrow}$. 

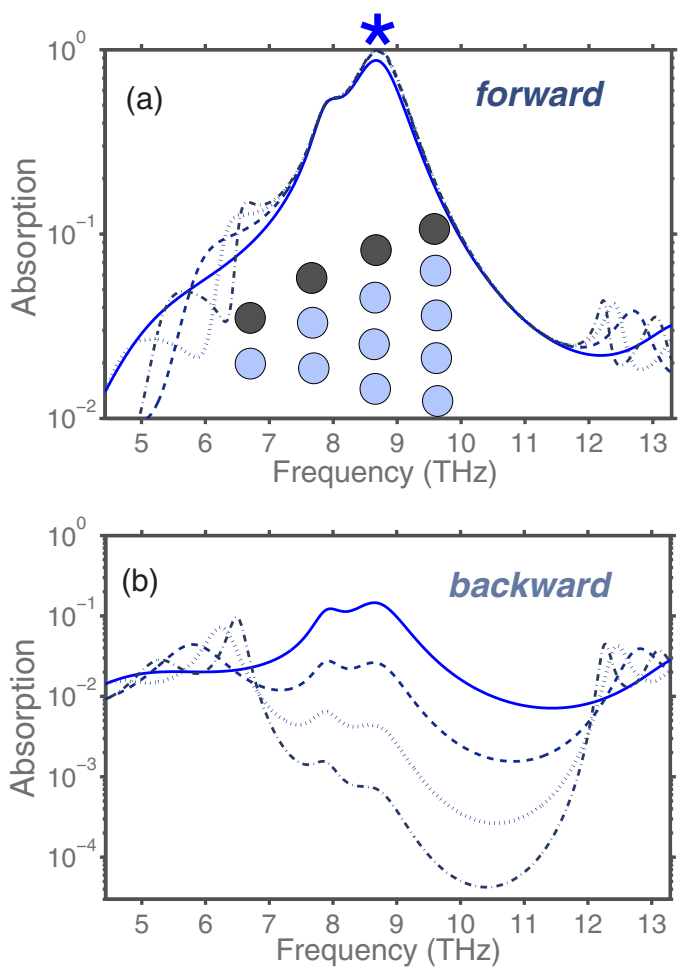

FIG. 4. (Color online) (a) $A^{\rightarrow}$ and (b) $A^{\leftarrow}$ vs frequency for four composite structures with $N=1$ : solid line $-M=1$, dashed line - $M=2$, dotted line - $M=3$, dash-dotted line - $M=4$; $d / a=0.4, \theta=0$; the lines for $M=2, M=3$, and $M=4$ coincide at the maximum in plot (a); four insets in plot (a) show geometry within a single period in the $x$ direction, for the structures with $M=1$, $M=2, M=3$, and $M=4$, from the left to the right.

$\operatorname{Re} \epsilon_{P} \approx 0.65$ should enable efficient penetration into and, thus, efficient absorption in the lossy material.

Thus, one-way absorption, with $A^{\rightarrow}=1$ and $A^{\leftarrow}=0$ can be obtained in rather thin rod-array based structures and does not require fully solid structures. In other words, large air gaps between the structural components are allowed. In Fig. 4, the frequency dependencies of $A^{\rightarrow}$ and $A^{\leftarrow}$ are presented for the four structures, which are the same as or similar to those in Fig. 3 and differ from each other in the value of $M$. As expected, $A^{\leftarrow}$ is decreased with increase of $M$. In particular, for $M=4$, absorption contrast $C_{A}=A^{\rightarrow} / A^{\leftarrow}>$ $10^{3}$ at $\max A^{\rightarrow}$ that occurs at $f=8.68 \mathrm{THz}$. To further increase $C_{A}$, structures with larger $M$ and, thus, with total thickness larger than $D=4 a+d$ are required.

Figure 5 presents $A \rightarrow$ and $C_{A}$ vs frequency for the structure from Figs. 3(c) and 3(d), at different values of $\theta$. Perfect absorption in the forward-incidence case and high forwardto-backward absorption contrast remain in a very wide range of variation in $\theta$. The difference in spectral locations of max $A^{\rightarrow}=1$ is just $0.4 \%$ for $\theta$ varying from 0 to $20^{\circ}$ and $1.6 \%$ for $\theta$ varying from 0 to $45^{\circ}$. Strong contrast is achieved at $\max A \rightarrow$ for $\theta=0$ that is even more enhanced with increasing $\theta$. In particular, at $\max A^{\rightarrow}, C_{A} \approx 230$ for $\theta=0$ and $C_{A} \approx 700$ for $\theta=45^{\circ}$. A further decrease of $C_{A}$ at both $\theta=0$ and $\theta>0$ can be obtained by increasing $M$, similarly to Fig. 4 . Note that the maxima of $A^{\rightarrow}$ and $C_{A}$ correspond to different frequencies.
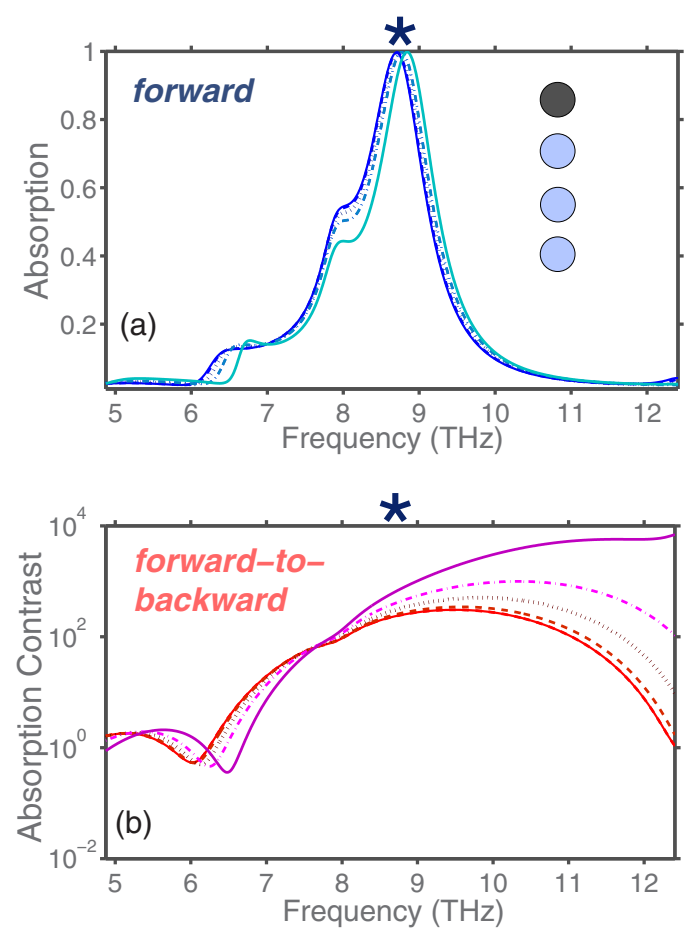

FIG. 5. (Color online) (a) $A^{\rightarrow}$ and (b) $C_{A}$ vs frequency for the composite structure with $M=3, N=1, d / a=0.4$. Left (a) and lower (b) solid line: $\theta=0$; dashed line: $\theta=10^{\circ}$; dotted line: $\theta=20^{\circ}$; dash-dotted line: $\theta=30^{\circ}$; right (a) and upper (b) solid line: $\theta=45^{\circ}$.

The question regarding their possible coincidence remains open.

From the obtained results (both shown and not shown), it follows that the lossy layer could be of a rather arbitrary shape, at least if $M$ is large enough to obtain a well pronounced stop band. Figure 6 presents $A^{\rightarrow}, A^{\leftarrow}, R^{\rightarrow}, R^{\leftarrow}$, and $T^{\rightarrow}=T^{\leftarrow}$ vs frequency for the two structures, which contain a finitethickness, thin, homogeneous layer made of GaAs and either one or three rod layers made of a nondispersive dielectric. In fact, these structures differ from those in Fig. 3 only in the geometry of the GaAs layer. Its thickness $t$ is chosen here so that the total volume occupied by GaAs is the same as in the all-rod structures, i.e., $t=\pi d^{2} /(4 a)$. The distance between the midplane of the GaAs layer and the closest rod axis is equal to $a$. One can see that all the basic features observed in Fig. 3 remain in Fig. 6. In particular, they include the regime of $A^{\rightarrow}=1$ and $A^{\leftarrow}=0$, which is observed in Figs. 6(c) and $6(\mathrm{~d})$ at $f \approx 8.68 \mathrm{THz}$. It is noteworthy that wide-band directional selectivity in absorption is obtained here owing to a stop band, which is only connected with the periodicity of the nondispersive part of the structure.

In Fig. 7, $A^{\rightarrow}$ and $A^{\leftarrow}$ vs frequency are shown for four structures that contain a thin solid GaAs layer and different number of the nondispersive rod layers. As in Fig. 4, two such layers are enough for obtaining of $A^{\rightarrow}=1$. However, on the contrary to Fig. 4, a larger value of $M$ is required in order to obtain the same and larger values of $C_{A}$. This might occur since the polar dielectric rods in Figs. 3 and 4 play not only the role of absorbers enhanced by reflections, but also enhance the stop band. Indeed, $\epsilon_{\mathrm{ND}}$ and $\epsilon_{P}(\omega)$ have quite close 

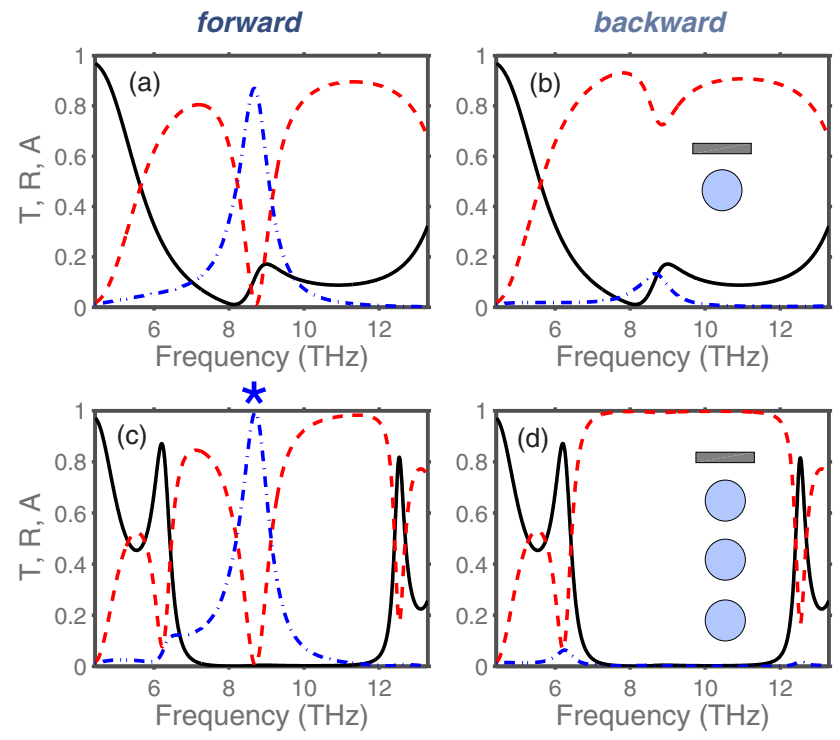

FIG. 6. (Color online) $T^{\rightarrow}, R^{\rightarrow}$, and $A^{\rightarrow}$ (a), (c) and $T^{\leftarrow}, R^{\leftarrow}$, and $A^{\leftarrow}$ (b), (d), for two composite structures with (a), (b) $M=1$ and homogeneous GaAs layer, and (c), (d) $M=3$ and homogeneous GaAs layer; $d / a=0.4, \theta=0$; solid line: $T^{\rightarrow}$ and $T^{\leftarrow}$; dashed line: $R^{\rightarrow}$ and $R^{\leftarrow}$; dash-dotted line: $A^{\rightarrow}$ and $A^{\leftarrow}$.

values at the stop band edges, because $\epsilon_{\mathrm{ND}}=\epsilon_{\infty}$ and the value of $d / a$ is kept. These features can be used as a basis of the general strategy to engineer structures containing polar and nondispersive dielectrics.
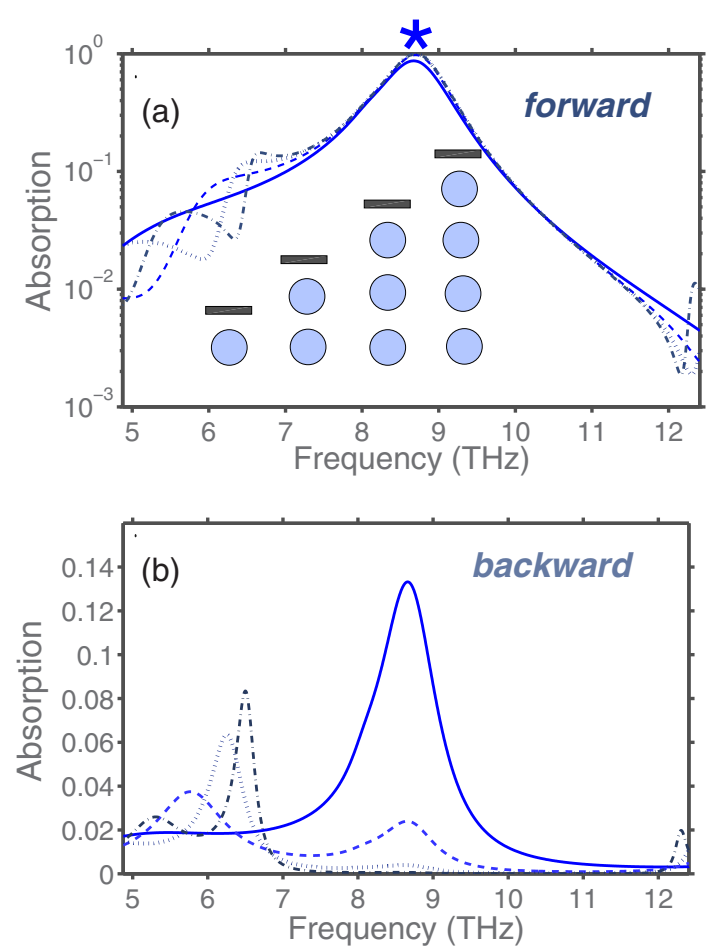

FIG. 7. (Color online) Same as Fig. 4 but for the $\mathrm{P}$ part that represents a homogeneous GaAs layer; four insets in plot (a) schematically show geometry of a single period for the structures with $M=1, M=2, M=3$, and $M=4$, from the left to the right.
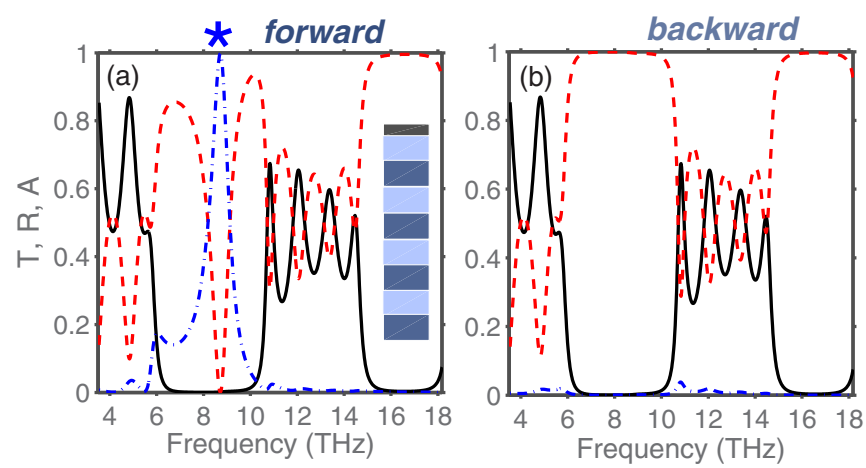

FIG. 8. (Color online) $T^{\rightarrow}, R^{\rightarrow}$, and $A^{\rightarrow}$ (a) and $T^{\leftarrow}, R^{\leftarrow}$, and $A^{\leftarrow}$ (b) for the multilayer composite structure with a homogeneous GaAs layer placed at the upper interface, $\theta=0$; solid line: $T^{\rightarrow}$ and $T^{\leftarrow}$; dashed line: $R^{\rightarrow}$ and $R^{\leftarrow}$; dash-dotted line: $A^{\rightarrow}$ and $A^{\leftarrow}$.

Since the directional selectivity has been observed in Figs. 2-7 when higher diffraction orders are evanescent, it can be expected that the above discussed effects may have an analog in nonsymmetric one-dimensional multilayer structures. Then, the possible contribution of the evanescent orders to absorption would entirely be avoided. Figure 8 presents $A \rightarrow$, $A^{\leftarrow}, R^{\rightarrow}, R^{\leftarrow}$, and $T^{\rightarrow}=T^{\leftarrow}$ vs frequency for the structure
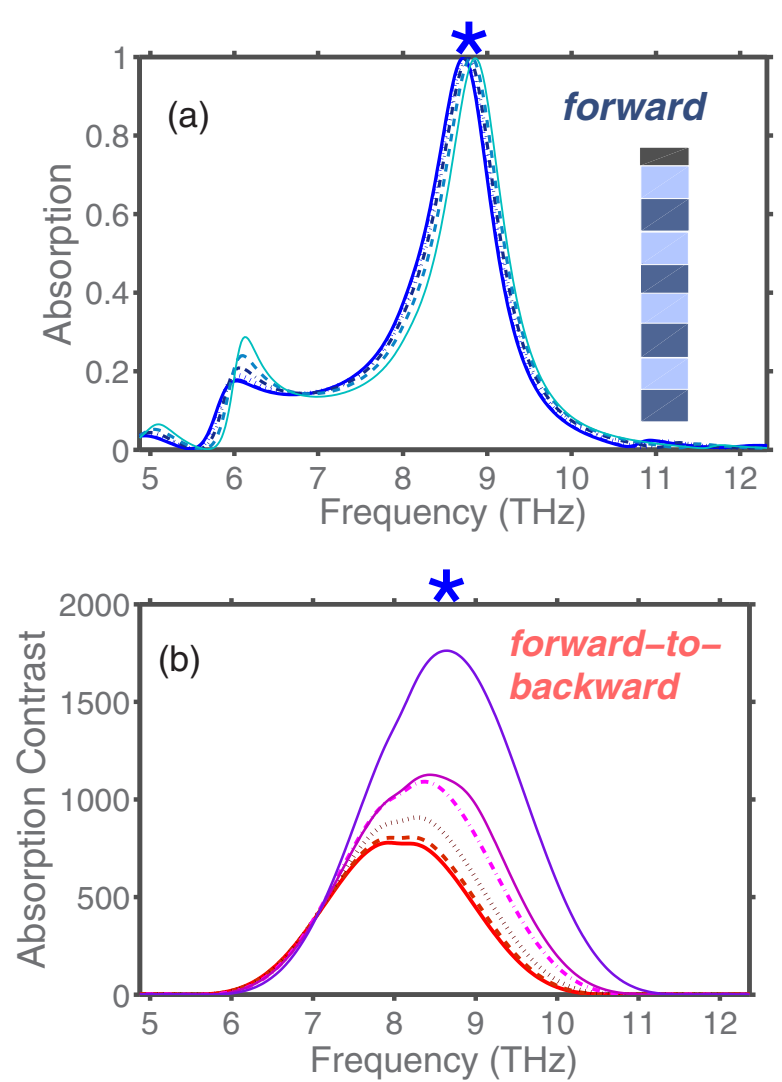

FIG. 9. (Color online) (a) $A^{\rightarrow}$ and (b) $C_{A}$ vs frequency for the composite multilayer structure in Fig. 8. Left (a) and lower (b) solid line: $\theta=0$; dashed line: $\theta=10^{\circ}$; dotted line: $\theta=20^{\circ}$; dash-dotted line: $\theta=30^{\circ}$; right (a) and second upper (b) solid line: $\theta=40^{\circ}$; uppermost solid line (b): $\theta=50^{\circ}$. 
that comprises four pairs of the alternating $\mathrm{SiO}_{2}(\epsilon=2.05)$ and Si $(\epsilon=12.25)$ layers, with the multilayer period $p=0.75 a$ and thickness of a $\mathrm{SiO}_{2}$ layer $q=0.4 p$, and a thin $\mathrm{GaAs}$ layer with thickness $t$ that is placed on the forward-incidence side, i.e., at the top of the most upper layer made of $\mathrm{SiO}_{2}$. Accordingly, the structure is terminated from the lower, i.e., backward-incidence side with a Si layer. The same thickness of the GaAs layer is chosen as in Fig. 6. Hence, the total thickness is $D=t+4 p$. As expected, the one-way absorption regime with $A^{\rightarrow}=1$ and $A^{\leftarrow}=0$ can be obtained in the multilayer structures. In Fig. 8, it appears at $f \approx 8.7 \mathrm{THz}$. Hence, one can decide between two-dimensional rod structures, onedimensional multilayer structures, and hybrid structures comprising rod and layer components, when additional reasons, e.g., those related to fabrication and integration, should be taken into account. As we see, all three types of the structures enable similar directional selectivity for absorption and reflection.

The frequency dependencies of $A^{\rightarrow}$ and $C_{A}$ at different values of $\theta$ are presented in Fig. 9 for the same structure as in Fig. 8. It is seen that the increase of $\theta$ results in the increase of max $C_{A}$. It exceeds $1.5 \times 10^{3}$ at the maximum of $A^{\rightarrow}$ when $\theta=50^{\circ}$. Higher contrasts are observed here as compared to the rod structure in Fig. 5. Moreover, the maxima of $C_{A}$ are now downshifted as compared to the maxima of $A^{\rightarrow}$. The larger the $\theta$, the weaker the shift is.

\section{CONCLUSIONS}

To summarize, low-profile, one- and two-dimensional structures enabling the perfect absorption of terahertz waves in the forward-incidence case and the perfect reflection in the backward-incidence case have been suggested, which contain GaAs, a polar dielectric, and a nondispersive lossless dielectric. The perfect absorption can be obtained in the structures both with and without air gaps between the structural components. For the all-rod performances, absorption is enhanced by reflections from the lossless part in the forward-incidence case, whereas stop band and reflection are enhanced in the backward-incidence case by the absorbing rod layer. For the performances containing a thin homogeneous absorbing layer, the enhancement of absorption by reflection from the lossless part takes place, too. Backing the absorbing layer, being either of a rod type or homogeneous, with a properly designed reflector can be more important for the perfect absorption than increasing the volume occupied by the absorbing material. This feature is expected to be in agreement with the recently developed theories of resonance absorption. It is interesting that the perfect absorption is obtained while the imaginary part of GaAs permittivity is far from the maximal value. Although consideration in this paper is restricted to $\mathrm{GaAs}$, other polar dielectrics, e.g., $\mathrm{LiF}$ and $\mathrm{NaCl}$ can be utilized for the same or similar mechanism of the perfect one-way absorption. The obtained results demonstrate high forward-to-backward absorption contrast that exceeds $10^{3}$ and indicate the routes to its further increase. Variation of the incidence angle in a wide range leads just to a weak spectral shift of the perfect absorption peak, whose location does not coincide with that of the contrast in the general case. This feature can be used, for instance, for wide-angle direction selective sensing. The neighboring frequency ranges may show high transmittance and, thus, enable other functionalities that can be combined with one-way absorber in one multifunctional device.

\section{ACKNOWLEDGMENTS}

This work is supported by the projects DPT-HAMIT, DPT-FOTON, and NATO SET-193, as well as TUBITAK under Projects No. 113E331, No. 109A015, and No. 109E301. The contribution of A.E.S. has partially been supported by the Matsumae International Foundation (Japan), TUBITAK in the framework of the Visiting Scientists Programme, and ESF in the framework of the RNP NEWFOCUS. E.O. acknowledges partial support from the Turkish Academy of Sciences.
[1] M. J. Lockyear, A. P. Hibbins, K. R. White, and J. R. Sambles, Phys. Rev. E 74, 056611 (2006).

[2] A. E. Serebryannikov, Phys. Rev. B 80, 155117 (2009).

[3] G. Shvets, Appl. Phys. Lett. 89, 141127 (2006).

[4] V. Yannopapas, Phys. Rev. A 88, 043837 (2013).

[5] R. Singh, E. Plum, C. Menzel, C. Rockstuhl, A. K. Azad, R. A. Cheville, F. Lederer, W. Zhang, and N. I. Zheludev, Phys. Rev. B 80, 153104 (2009).

[6] M. Beruete, A. E. Serebryannikov, V. Torres, M. Navarro-Cia, and M. Sorolla, Appl. Phys. Lett. 99, 154101 (2011).

[7] A. E. Serebryannikov, E. Ozbay, and S. Nojima, Opt. Express 22, 3075 (2014).

[8] C. Menzel, C. Helgert, C. Rockstuhl, E.-B. Kley, A. Tünnermann, T. Pertsch, and F. Lederer, Phys. Rev. Lett. 104, 253902 (2010).

[9] S. Zhang, F. Liu, T. Zentgraf, and J. Li, Phys. Rev. A 88, 023823 (2013).
[10] S. Cakmakyapan, H. Caglayan, A. E. Serebryannikov, and E. Ozbay, Appl. Phys. Lett. 98, 051103 (2011).

[11] M. Stolarek, M. Yavorskiy, R. Kotynski, C. J. Zapata Rodriguez, J. Lusakowski, and T. Szoplik, Opt. Lett. 38, 839 (2013).

[12] V. Kuzmiak and A. A. Maradudin, Phys. Rev. A 86, 043805 (2012).

[13] D. Jalas, A. Petrov, M. Eich, W. Freude, S. Fan, Z. Yu, R. Baets, M. Popovic et al., Nat. Photon. 7, 579 (2013).

[14] R. J. Potton, Rep. Prog. Phys. 67, 717 (2004).

[15] A. Armitage, M. S. Skolnick, A. V. Kavokin, D. M. Whittaker, V. N. Astratov, G. A. Gehring, and J. S. Roberts, Phys. Rev. B 58, 15367 (1998).

[16] G. S. Agarwal and S. Dutta Gupta, Opt. Lett. 27, 1205 (2002).

[17] N. I. Landy, S. Sajuyigbe, J. J. Mock, D. R. Smith, and W. J. Padilla, Phys. Rev. Lett. 100, 207402 (2008).

[18] N. I. Landy, C. M. Bingham, T. Tyler, N. Jokerst, D. R. Smith, and W. J. Padilla, Phys. Rev. B 79, 125104 (2009). 
[19] G. Dayal and S. A. Ramakrishna, Opt. Express 20, 17503 (2012).

[20] W. Wu and R. Magnusson, Opt. Lett. 37, 2103 (2012).

[21] Q. Feng, M. Pu, C. Hu, and X. Luo, Opt. Lett. 37, 2133 (2012).

[22] J. W. Cleary, R. Soref, and J. R. Hendrickson, Opt. Express 21, 19363 (2013).

[23] S. Thongrattanasiri, F. H. L. Koppens, and F. J. Garcia de Abajo, Phys. Rev. Lett. 108, 047401 (2012).

[24] M. J. Lockyear, A. P. Hibbins, J. R. Sambles, P. A. Hobson, and C. R. Lawrence, Appl. Phys. Lett. 94, 041913 (2009).

[25] D. Liang, Y. Huo, Y. Kang, K. X. Wang, A. Gu, M. Tan, Z. Yu et al., Adv. Energy Mater. 2, 1254 (2012).

[26] C. Argyropoulos, K. Q. Le, N. Mattiucci, G. D’Aguanno, and A. Alu, Phys. Rev. B 87, 205112 (2013).

[27] K. Aydin, V. E. Ferry, R. M. Briggs, and H. A. Atwater, Nat. Commun. 2, 517 (2011).

[28] P. N. Bartlett, Y. Sugawara, and J. J. Baumberg, Nat. Photon. 2, 299 (2008).

[29] N. Bonod and E. Popov, Opt. Lett. 33, 2398 (2008).

[30] Y. Avitzour, Y. A. Urzhumov, and G. Shvets, Phys. Rev. B 79, 045131 (2009).
[31] M. A. Kats, R. Blanchard, P. Genevet, and F. Capasso, Nat. Mater. 12, 20 (2013).

[32] H.-T. Chen, Opt. Express 20, 7165 (2012).

[33] M. A. Kats, S. J. Byrnes, R. Blanchard, M. Kolle, P. Genevet, J. Aizenberg, and F. Capasso, Appl. Phys. Lett. 103, 101104 (2013).

[34] M. M. Sigalas, C. M. Soukoulis, C. T. Chan, and K. M. Ho, Phys. Rev. B 49, 11080 (1994).

[35] P. B. Catrysse and S. Fan, Phys. Rev. B 75, 075422 (2007).

[36] S. Nojima, Phys. Rev. B 57, R2057(R) (1998).

[37] S. Foteinopoulou, M. Kafesaki, E. N. Economou, and C. M. Soukoulis, Phys. Rev. B 84, 035128 (2011).

[38] M. Kafesaki, A. A. Basharin, E. N. Economou, and C. M. Soukoulis, Photon. Nanostruct.: Fund. Appl. 12, 376 (2014).

[39] V. Yannopapas, Phys. Rev. B 75, 035112 (2007).

[40] T. Magath and A. E. Serebryannikov, J. Opt. Soc. Am. A 22, 2405 (2005).

[41] C. Kittel, Introduction to Solid State Physics (John Wiley and Sons, Hoboken, NJ, 2005). 